\title{
Modelling and Prediction of 25-Hydroxyvitamin D Levels in Norwegian Relapsing-Remitting Multiple Sclerosis Patients
}

\author{
J. Šaltytė Benth ${ }^{a, c} \quad$ K.-M. Myhrd, e K.I. Løken-Amsrud ${ }^{f} \quad$ A.G. Beiske ${ }^{g}$ \\ K.S. Bjerve ${ }^{\text {h, j }}$ H. Hovdal ${ }^{i}$ R. Midgard ${ }^{k}$ T. Holmøy ${ }^{b, c}$
}

${ }^{\mathrm{a}} \mathrm{H} \varnothing \mathrm{KH}$, Research Centre and b ${ }^{\mathrm{b}}$ Department of Neurology, Akershus University Hospital, Lørenskog, ${ }^{\mathrm{C}}$ Institute of Clinical Medicine, Campus Ahus, University of Oslo, Oslo, ${ }^{\mathrm{d}}$ The Norwegian Multiple Sclerosis Competence Centre, Department of Neurology, Haukeland University Hospital, and ${ }^{\mathrm{E}} \mathrm{KG}$-Jebsen MS-Research Centre, Department of Clinical Medicine, University of Bergen, Bergen, ${ }^{\mathrm{f} D e p a r t m e n t}$ of Neurology, Innlandet Hospital Trust, Lillehammer, g MS Centre Hakadal, Hakadal, Departments of hedical Biochemistry and 'Neurology, St. Olavs Hospital, Trondheim University Hospital, and 'Department of Laboratory Medicine, Children's and Womens's Health, Norwegian University of Science and Technology, Trondheim, and kDepartment of Neurology, Molde Hospital, Molde, Norway

\section{Key Words}

25-Hydroxyvitamin D • Multiple sclerosis • Multivariate model $\cdot$ Seasonal fluctuation $\cdot$ Prediction

\begin{abstract}
Background/Aim: 25-Hydroxyvitamin D (25(OH)D) levels are suggested to influence the susceptibility and risk of disease progression in multiple sclerosis (MS). Seasonal fluctuation of $25(\mathrm{OH}) \mathrm{D}$ levels may differ in magnitude between individuals. The purpose of this paper was to model the seasonal fluctuation of vitamin D in Norwegian MS patients and to examine to which extent one single $25(\mathrm{OH}) \mathrm{D}$ measurement predicts the level at other time points throughout the year. Methods: During December 2004 and July 2008, 762 serum samples were collected from 92 Norwegian relapsing-remitting MS patients. Time series analysis and multivariate modelling techniques were used to model seasonal fluctuations and intra- and inter-individual variations in 25(OH)D values. Results: Most patients reached their lowest 25(OH)D level in March/April and the highest in July/August. There were substantial intra-individual variations in the extent of seasonal fluctuation, with $36.6 \%$ of explainable varia-
\end{abstract}

tion in seasonally adjusted 25(OH)D levels (on a logarithmic scale) attributable to the patient level. The remaining $63.4 \%$ could be accounted for by sources of inter-individual variation. Both the total and inter-individual variabilities were lowest in February, and the prediction interval in this month was up to $26 \%$ narrower compared to other months. The prediction intervals would be at least $21 \%$ wider with only one observation available per patient. Conclusions: The seasonal fluctuations of 25(OH)D levels in Norwegian relapsing-remitting MS patients are subject to pronounced intra- and inter-individual variation. The most representative measurements of 25(OH)D levels are taken in February.

Copyright $\odot 2012$ S. Karger AG, Basel

\section{Introduction}

Vitamin D exerts immunomodulatory and possibly also neuroprotective properties that are potentially relevant in multiple sclerosis (MS) [1]. The epidemiological evidence for vitamin D involvement in MS includes prospective studies showing that people with 25-hydroxyvitamin D (25(OH)D) levels above $100 \mathrm{nmol} / \mathrm{l}$ are less like-

\section{KARGER}

Fax +41613061234

E-Mail karger@karger.ch

www.karger.com (c) 2012 S. Karger AG, Basel

0251-5350/12/0392-0084\$38.00/0

Accessible online at:

www.karger.com/ned
Jūratė Šaltytė Benth

Research Centre, Akershus University Hospital

PB 95

NO-1478 Lørenskog (Norway)

Tel. +47 6796 8723, E-Mail jurates@ medisin.uio.no 
ly to develop MS later in life than those with levels lower than $50 \mathrm{nmol} / \mathrm{l}$ [2], and that a high intake of vitamin D supplements at baseline protect against subsequent MS development [3]. Moreover, observational studies including one or a few, often deseasonalised, vitamin D measurements from each patient have suggested that a poor vitamin $\mathrm{D}$ status is associated with an unfavourable course of MS [4-8]. In most individuals, ultraviolet B (UVB) radiation from sun exposure is the main source of vitamin $\mathrm{D}[9,10]$. However, in areas with temperate climate, the amount of UVB exposure during the winter is insufficient to induce synthesis of vitamin $\mathrm{D}$ in the human skin [11], thus vitamin D levels are subject to seasonal fluctuation. The extent and individual variation of the seasonal fluctuation of vitamin D levels are not fully known and depend on several factors including diet, latitude, climate, sun habits, skin colour, age, and body composition [9]. Notably, both genome-wide association studies and twin studies of MS patients have shown that vitamin D levels are under genetic control [12, 13], further suggesting that the extent of seasonal fluctuation of vitamin $\mathrm{D}$ levels may differ between subjects.

Due to its relatively long half-life, $25(\mathrm{OH}) \mathrm{D}$ is used as a measure of the vitamin D status in the body. With only one or a few measurements available from each patient, it is difficult, maybe even impossible, to quantify the individual fluctuation of $25(\mathrm{OH}) \mathrm{D}$ levels. Therefore, it is not known to which extent a single $25(\mathrm{OH}) \mathrm{D}$ measurement reflects the concentration at other time points, and thus the individual's overall vitamin D status.

The purpose of this study was to model the seasonal fluctuation of vitamin $\mathrm{D}$ in Norwegian MS patients and to examine to what extent one single $25(\mathrm{OH}) \mathrm{D}$ value predicts the $25(\mathrm{OH}) \mathrm{D}$ level at other time points throughout the year. To address this question, we used a data set comprising up to nine repeated 25(OH)D measurements from 92 Norwegian relapsing-remitting MS patients who had participated in a randomised clinical trial of omega- 3 fatty acids in MS (the OFAMS study [14]), collected throughout a period of 24 months, to build a model incorporating both seasonal fluctuations and individual variations in $25(\mathrm{OH}) \mathrm{D}$ values. The suggested model is implemented in a spreadsheet (provided as an online supplement in Microsoft Excel; for all online supplementary material, see www. karger.com/doi/10.1159/000339360) and can be used to predict the monthly values of $25(\mathrm{OH}) \mathrm{D}$ for an individual patient based on one single measurement and estimate the accuracy of the prediction through the corresponding prediction intervals.

Modelling and Prediction of 25(OH)D

Levels in MS Patients

\section{Patients and Methods}

\section{Study Population and Design}

The inclusion criteria of the OFAMS study [15] comprised patients with relapsing-remitting MS according to the McDonald criteria, aged 18-58 years, Expanded Disability Status Scale score of 5.5 or less at inclusion, and at least one relapse or one new $\mathrm{T}_{1}$ weighted gadolinium-enhancing lesion or one new $\mathrm{T}_{2}$-weighted lesion on MRI in the year prior to inclusion. All 92 patients included in the OFAMS study ( 60 women and 32 men, all Caucasian, median age at inclusion 39 years (range 19-58), median Expanded Disability Status Scale score at inclusion 2.0 (range 0.0-4.0), median time from first symptom 3 years (range $0-23)$ ) were included in the present study. The participants received either omega- 3 fatty acids (Triomar ${ }^{\mathrm{TM}}$; Pronova Biocare, Sandefjord, Norway) or placebo (corn-oil) during the whole study period. After 6 months, all participants received interferon- $\beta$-1a (Rebif ${ }^{\mathrm{TM}}$; Serono International, Geneva, Switzerland). None of the treatments contained vitamin $\mathrm{D}$. The participants were instructed not to take any other omega-3 supplement. Otherwise, no particular advice regarding diet or supplementation with vitamin D was given. Information on UVB exposure or vitamin D supplementation was not recorded.

\section{Measuring of $25(\mathrm{OH}) \mathrm{D}$}

$25(\mathrm{OH}) \mathrm{D}$ blood samples were collected between December 2004 and July 2008 by venepuncture consecutively at the baseline visit and then at visits at $1,3,6,7,9,12,18$, and 24 months [15]. After centrifugation, serum samples were stored at $-80^{\circ} \mathrm{C}$ until analysis, which was performed simultaneously for all samples from each patient. $25(\mathrm{OH}) \mathrm{D}$ was measured by radioimmunoassay (RIA kit; ImmunoDiagnostic Systems, Boldon, UK) at the Department of Medical Biochemistry, St. Olavs Hospital, Trondheim, Norway. The coefficient of variation was $5.4 \%$ at $29 \mathrm{nmol} / \mathrm{l}$ and $6.3 \%$ at $112 \mathrm{nmol} / \mathrm{l}$.

\section{Exploratory Data Analysis}

Available data were 25(OH)D measurements from 92 patients at 1-9 time points, yielding 774 observations producing repeated monthly time series for 43 months. Two patients comprising 12 individual observations were excluded from further analyses and used in model validation.

Descriptive statistics for $25(\mathrm{OH}) \mathrm{D}$ are presented in table 1. Analyses showed a skewed distribution of $25(\mathrm{OH}) \mathrm{D}$ levels, confirmed by a skewness coefficient of 1.20. Kurtosis was rather high, supporting the deviation from normality. In addition, we had highly varying $25(\mathrm{OH}) \mathrm{D}$ values (fig. 1a). We therefore chose to logarithmically transform the $25(\mathrm{OH}) \mathrm{D}$ (denoted log $25(\mathrm{OH}) \mathrm{D}$ ) levels. This transformation symmetrised the distribution well (fig. 1b). The skewness and kurtosis became essentially zero (table 1), and mean and median almost equal.

A repeated time series plot (fig. 1c) demonstrated a clear seasonal pattern with the highest $\log 25(\mathrm{OH}) \mathrm{D}$ levels in late summer/ early autumn and the lowest in early spring. This coincided with results from previous studies in patients with MS [6, 7] and healthy individuals $[16,17]$. The inter-individual variation in $25(\mathrm{OH}) \mathrm{D}$ levels was highest in the late summer and much lower during the winter/early spring (see table 2 for descriptives). Clearly, the logarithmic transformation stabilized the variation, even though the hypothesis of homogeneity of total (sum of inter- and 
Fig. 1. Histogram of $25(\mathrm{OH}) \mathrm{D}$ levels (a), histogram of $\log 25(\mathrm{OH}) \mathrm{D}$ levels (b), log $25(\mathrm{OH}) \mathrm{D}$ levels with fitted seasonal component (c), and seasonally adjusted log 25(OH)D levels $(\mathbf{d})$.

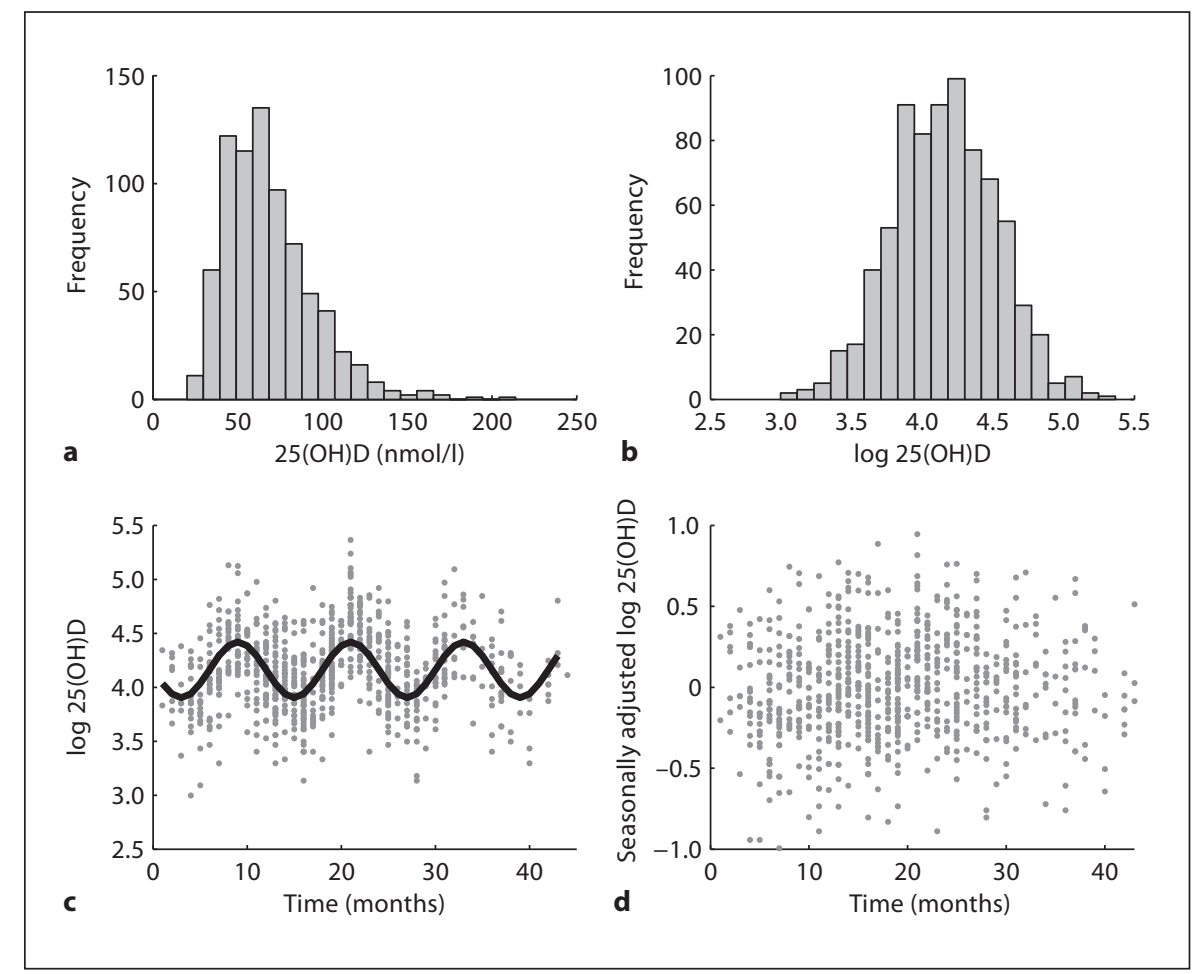

intra-individual) variances at different months was rejected by Levene's test $(\mathrm{p}<0.001)$.

The average maximum-minimum ratio for $\log 25(\mathrm{OH}) \mathrm{D}$ values was 1.2, with a standard deviation of 0.1 and a range of 1.0-1.4, indicating some inter-variability in patients' $\log 25(\mathrm{OH}) \mathrm{D}$ levels. The intra-class correlation coefficient (ICC) is defined as a ratio of the inter-individual variance $S D_{B}^{2}$ to the total variance $S D_{B}^{2}+$ $S D_{W}^{2}\left(S D_{W}^{2}\right.$ being the intra-individual variance), i.e. $S D_{B}^{2} /\left(S D_{B}^{2}+\right.$ $S D_{W}^{2}$ ), and shows which proportion of the total variance occurs between patients. The ICC differed considerably between months, being lowest in February and highest in July (table 2). The ICC for the whole study period shows that $63.4 \%$ of the variability in $\log$ $25(\mathrm{OH}) \mathrm{D}$ levels was reflected by inter-individual differences, whereas $36.6 \%$ can be attributed to intra-individual variations. Such a clustering effect implies that a patient having log $25(\mathrm{OH}) \mathrm{D}$ levels well above the average of the whole patient group at one specific month would most likely remain higher than the average throughout the year, and vice versa. Moreover, the differences between $\log 25(\mathrm{OH}) \mathrm{D}$ values taken 12 months apart from each other were explored. Although the average differences between log 25(OH)D levels at baseline and 12 months later were significantly different from zero ( $p=0.02$, $t$ test), the average differences between $\log 25(\mathrm{OH}) \mathrm{D}$ values at months 6 and 18, and months 12 and 24 were not significantly different from zero, demonstrating that the individual $\log 25(\mathrm{OH}) \mathrm{D}$ levels are quite stable over time.

Time Series Model for $\log 25(\mathrm{OH}) \mathrm{D}$ Levels

Our primary goal was to build a model able to predict the 25(OH)D levels at 11 time points (months) conditioned on one observation available. We first modelled and estimated the time
Table 1. Descriptive statistics for 25(OH)D levels, log 25(OH)D levels, and the final residuals of 762 samples

\begin{tabular}{lcll}
\hline Characteristics & $25(\mathrm{OH}) \mathrm{D}$ levels & $\begin{array}{l}\log 25(\mathrm{OH}) \mathrm{D} \\
\text { levels }\end{array}$ & $\begin{array}{l}\text { Final } \\
\text { residuals }\end{array}$ \\
\hline Min. & $20^{\mathrm{a}}$ & 3.00 & -3.32 \\
Max. & $214^{\mathrm{a}}$ & 5.37 & 3.05 \\
Mean $\pm \mathrm{SD}$ & $68.34 \pm 26.15^{\mathrm{a}}$ & $4.16 \pm 0.37$ & $0.02 \pm 1.03$ \\
Median & $63.00^{\mathrm{a}}$ & 4.14 & -0.01 \\
$\quad \mathrm{Q}_{1}, \mathrm{Q}_{3}{ }^{1}$ & $49.00,82.00^{\mathrm{a}}$ & $3.89,4.41$ & $-0.67,0.72$ \\
Skewness & 1.20 & 0.03 & 0.001 \\
Kurtosis & 2.47 & -0.02 & 0.03 \\
\hline
\end{tabular}

${ }^{\text {a }}$ Values are given in $\mathrm{nmol} / \mathrm{l}$.

${ }^{1} \mathrm{Q}_{1}$ and $\mathrm{Q}_{3}$ denote the first and the third quartiles.

series of monthly $\log 25(\mathrm{OH}) \mathrm{D}$ levels. In a good time series model, residuals obtained after removing deterministic components such as trend and periodic variations should be normally distributed, not autocorrelated, and have mean zero and homogeneous variance over time, i.e. be a so-called white noise. One of the often used time series modelling approaches is the decomposition method, where the time series are decomposed into a sum (sometimes a product) of several components: trend (long-term tendency) and seasonal (systematic), cyclic (repeated but irregular), and irregular (unsystematic short-time) variations. The different 
Table 2. Monthly mean values, inter- and intra-individual standard deviations of $25(\mathrm{OH}) \mathrm{D}$ and $\log 25(\mathrm{OH}) \mathrm{D}$ levels, and total standard deviation and ICC of log 25(OH)D levels

\begin{tabular}{|c|c|c|c|c|c|}
\hline \multirow[t]{2}{*}{ Month } & \multirow[t]{2}{*}{ Patients $(k)$} & \multirow{2}{*}{$\begin{array}{l}\text { Mean } 25(\mathrm{OH}) \mathrm{D} \\
\left(S D_{B}, S D_{W}\right), \mathrm{nmol} / \mathrm{l}\end{array}$} & \multicolumn{3}{|l|}{$\log 25(\mathrm{OH}) \mathrm{D}$} \\
\hline & & & mean $\left(S D_{B}, S D_{W}\right)$ & $\sqrt{S D_{B}^{2}+S D_{W}^{2}}$ & ICC \\
\hline January & $43(3)$ & $60.4(13.6,11.2)$ & $4.06(0.21,0.21)$ & 0.30 & 48.3 \\
\hline February & $40(3)$ & $57.0(7.4,14.4)$ & $4.01(0.14,0.24)$ & 0.28 & 26.3 \\
\hline March & $54(4)$ & $49.7(12.0,11.0)$ & $3.86(0.25,0.20)$ & 0.32 & 60.8 \\
\hline April & $28(3)$ & $53.5(21.8,6.5)$ & $3.92(0.34,0.11)$ & 0.36 & 89.9 \\
\hline May & $46(3)$ & $62.8(14.6,12.2)$ & $4.10(0.24,0.17)$ & 0.30 & 66.7 \\
\hline June & $56(4)$ & $72.7(16.5,14.1)$ & $4.24(0.25,0.17)$ & 0.30 & 67.4 \\
\hline July & $34(3)$ & $84.4(25.6,6.8)$ & $4.40(0.27,0.08)$ & 0.29 & 91.5 \\
\hline August & $52(3)$ & $100.5(30.0,15.1)$ & $4.56(0.26,0.17)$ & 0.31 & 71.1 \\
\hline September & $40(3)$ & $82.6(13.0,18.5)$ & $4.37(0.16,0.24)$ & 0.29 & 29.4 \\
\hline October & $35(3)$ & $72.1(18.1,19.3)$ & $4.21(0.29,0.23)$ & 0.37 & 60.4 \\
\hline November & $41(3)$ & $69.3(21.0,12.4)$ & $4.18(0.30,0.18)$ & 0.35 & 75.0 \\
\hline December & $56(3)$ & $61.9(17.9,11.0)$ & $4.07(0.29,0.17)$ & 0.34 & 74.6 \\
\hline Total & $90(9)$ & $68.3(16.2,20.6)$ & $4.16(0.25,0.19)$ & 0.32 & 63.4 \\
\hline
\end{tabular}

$k=$ Maximum number of measurements per patient; $S D_{B}, S D_{W}=$ inter-, intra-individual standard deviation; $\sqrt{S D_{B}^{2}+S D_{W}^{2}}=$ total standard deviation.

components are then estimated one by one and eliminated from the data. At each step, the autocorrelation function (ACF) and histogram are examined and descriptive statistics calculated to assess if there are still unmodelled components left in the model. The ACF is used to detect non-randomness (persistence) in a time series.

We denote the $\log 25(\mathrm{OH}) \mathrm{D}$ level for $i$-th patient at $j$-th occasion by $D_{i j}$, and each $D_{i j}$ is observed $n_{i}$ times at time $t_{i j}, i=1, \ldots, 92$, $j=1, \ldots, 43$. The average $\log 25(\mathrm{OH}) \mathrm{D}$ levels calculated for all patients at each time point were used for modelling the deterministic components. We decomposed the average log 25(OH)D level at time moment $j, D_{j}$, into a sum of mean component $S_{j}$, describing seasonal and other possible cyclic variations, and a residual component $\varepsilon_{i j}$, modelling the fluctuations around the mean, i.e.:

$$
D_{j}=S_{j}+\varepsilon_{i j} \text {. }
$$

We did not expect any time trend in $\log 25(\mathrm{OH}) \mathrm{D}$ levels due to the short time span. Moreover, detailed analyses showed no cyclic effects in the data. Seasonal variations appearing in regularly spaced peaks were modelled with a simple sine function:

$$
S_{j}=a_{1}+a_{2} \sin \left(\frac{2 \pi j}{12}\right) \text {. }
$$

The parameter $a_{1}$ describes the average level of $\log 25(\mathrm{OH}) \mathrm{D}$, and $a_{2}$ is the amplitude in seasonal variations.

As discussed earlier, the residuals of a time series model that fits data well should be white noise. However, because some heterogeneity might be left in the data after eliminating the deterministic components, we assumed that the residual process can be factorized into

$$
\varepsilon_{i j}=\sigma_{j} \epsilon_{i j}
$$

Modelling and Prediction of 25(OH)D

Levels in MS Patients with $i=1, \ldots, 92, j=1, \ldots, 43$, where $\sigma_{j}$ is a non-random (possibly) seasonal function meant to capture potential heterogeneity in the residuals, and $\epsilon_{i j}$ is assumed to be white noise. The function $\sigma_{j}$ could be estimated by exploiting the consequences of repeated observations at each time point.

\section{Multivariate Model}

One could consider to use only a seasonal component representing the seasonal average of $\log 25(\mathrm{OH}) \mathrm{D}$ levels for predicting an individual patient's levels throughout a year. However, the observed clustering within a patient in seasonally adjusted data clearly indicates the need for a model able to account for this clustering effect.

After fitting the time series model to the average log $25(\mathrm{OH}) \mathrm{D}$ levels, we were left with the residuals exhibiting white noise properties. Thus, we could ignore the time direction and reorder the residuals from the original time scale to 12 points along the line. We denote the residuals obtained after fitting the time series model by $R_{i k}$ for an $i$-th patient on the $k$-th occasion, $i=1, \ldots, 92, k=1, \ldots, 12$. Notably, the residuals (seasonally adjusted data) $R_{i k}$ are not averaged but observed $n_{i}$ times at time $t_{i k}$. Even though they are uncorrelated in time, due to clustering effect in data they will exhibit some correlations, which must be taken into account.

To be able to make monthly predictions conditioned on one observation for a certain individual, we needed a multivariate model gathering the information from all 12 points. The most natural was to assume that the 12 -variate distribution of residuals is normal. The marginal and conditional distributions derived from a multivariate normal distribution are also normal [18]. The prediction was therefore calculated as conditional mean, and conditional variance was used for building the prediction interval 
Fig. 2. ACF of $\log 25(\mathrm{OH}) \mathrm{D}$ levels (a), ACF of residuals of $\log 25(\mathrm{OH}) \mathrm{D}$ levels obtained after fitting the time series model (b), and empirical standard deviations of seasonally adjusted $\log 25(\mathrm{OH}) \mathrm{D}$ levels (c, lefthand axis, solid line) and of final residuals (c, right-hand axis, dashed line).

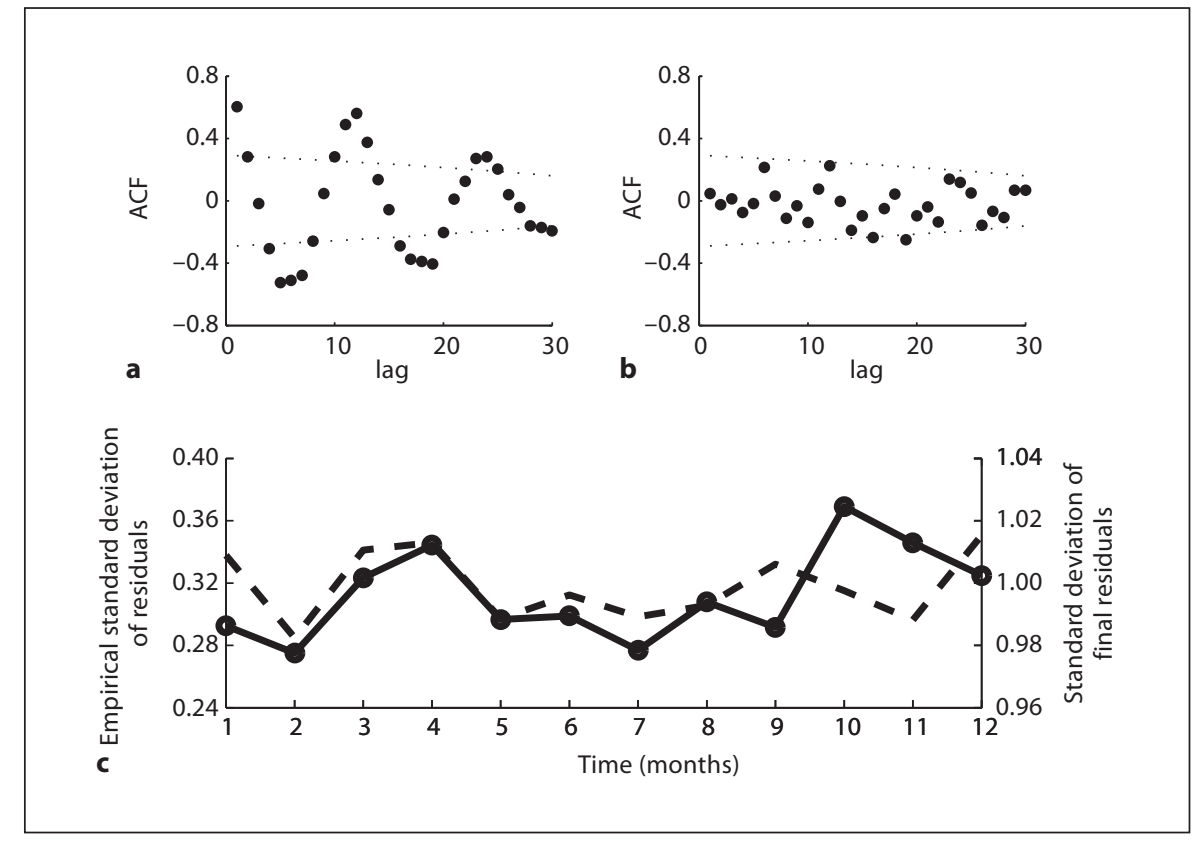

(PI). The mean and variance of $D_{i k}$ at any point $k$ conditioned on the observation at point $l(k, l=1, \ldots, 12)$ can, respectively, be calculated as [18]:

$$
E\left(D_{i k} / D_{i l}\right)=S_{k}+\sigma_{k}\left(D_{i l}-S_{l}\right)
$$

and

$$
\operatorname{var}\left(D_{i k} / D_{i l}\right)=\sigma_{k}^{2}\left(1-\rho_{k l}^{2}\right) .
$$

Here, $S_{k}$ is an average $\log 25(\mathrm{OH}) \mathrm{D}$ level to be predicted at point $k, \sigma_{k}^{2}=\operatorname{var}\left(R_{k}\right)$ is the variance of the residuals at the point $k, \sigma_{k}$ is the corresponding standard deviation, and $\rho_{k l}=\operatorname{corr}\left(R_{k}\right.$, $\left.R_{l}\right)$ is the correlation between the paired residuals at the points $k$ and $l, k, l=1, \ldots, 12$. By using the empirical variances of seasonally adjusted data at each time point and calculating the empirical correlations for all unique distances (differences between any two points on the 12-point line) and fitting a certain parametric function to them, we defined $\sigma_{k}^{2}$ and $\rho_{k l}$. Notably, the conditional variance $\operatorname{var}\left(D_{i k} / D_{i l}\right)$ can generally be patient dependent. However, the estimation of individual variances $\left(\sigma_{i k}^{2}\right)$ requires an amount of observations for each patient which might be difficult to attain in practice.

\section{Results}

\section{Seasonal component $S_{j}$}

The ACF of log 25(OH)D levels (fig. 2a) revealed seasonally varying autocorrelations far off the $95 \%$ confidence bands (significantly different from zero autocorrelations according to the Ljung-Box statistics), consistent with seasonality in the data. We had almost a 4-year time span, consisting of 43 time points, for estimating a seasonal component $S_{j}, j=1, \ldots, 43$. We fitted a simple sine function to the averages of replicates of $\log 25(\mathrm{OH}) \mathrm{D}$ levels at 43 time points. The parameters were estimated to be $a_{1}=4.16$ and $a_{2}=-0.26$, both significantly different from zero. The estimated seasonal component described the seasonal pattern well (fig. 1c). The seasonal function containing both sine and cosine terms (as in [19]) was also fitted. However, the differences were minor, and a simpler function was therefore chosen. Following the decomposition approach, we eliminated seasonal variations by subtracting the estimated seasonal component from the original observations (fig. 1d). Such seasonal adjustment was needed to make the true behaviour of time series more apparent.

The seasonally adjusted averages of $\log 25(\mathrm{OH}) \mathrm{D}$ values were not autocorrelated in time according to the Ljung-Box test (see also fig. 2b), indicating that there was no cyclic component in the averaged data. We therefore ignored the time direction and merged them from 43 to 12 time points along the line, where only the distance but not the direction counts. The seasonally adjusted log $25(\mathrm{OH}) \mathrm{D}$ values at each of the 12 points followed the normal distribution ( $\mathrm{p}>0.67$; Kolmogorov-Smirnov test). Note that the normality check and most other tests would not be possible on the 43-point axis due to few observations at each point, while gathering the data into 12 points gives us enough information. 


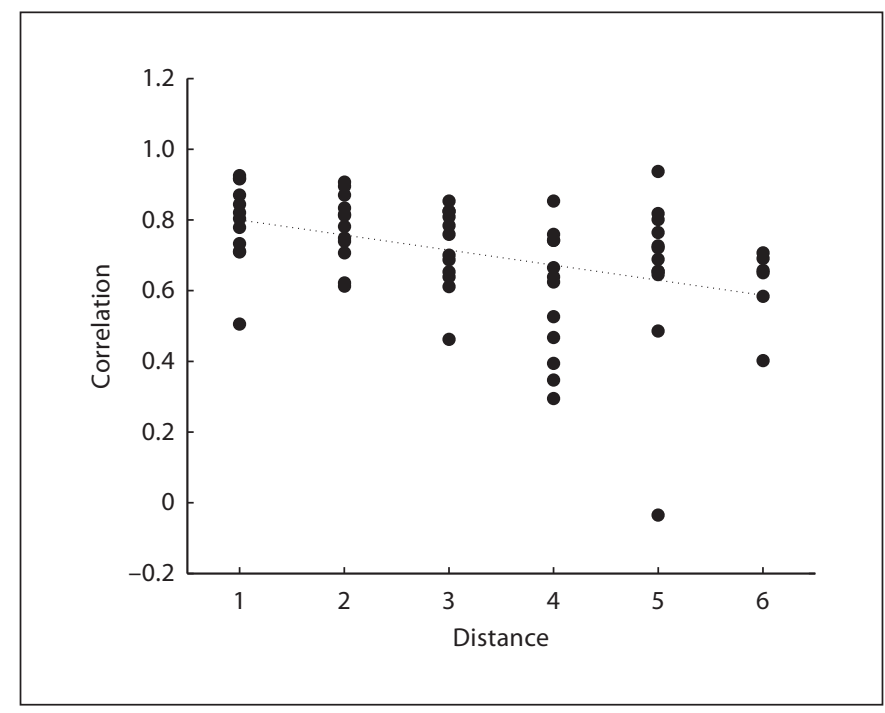

Fig. 3. Empirical correlations between pair-wise seasonally adjusted $\log 25(\mathrm{OH}) \mathrm{D}$ levels at different points and fitted correlation function (dotted line).

\section{Residuals}

As argued above, we could ignore the time direction in seasonally adjusted data. We observed some signs of heterogeneity in the seasonally adjusted $\log 25(\mathrm{OH}) \mathrm{D} \mathrm{lev}$ els (fig. 2c). Levene's test for homogeneity of variances did not give any statistical evidence of significantly different variances $(p=0.376)$. However, to obtain the residuals exhibiting white noise properties, we nevertheless chose to model them. This minor time dependency in the residuals was removed by dividing the residuals for different time points by the corresponding empirical standard deviation. In the presence of longer time series and clearer heterogeneous effects, one could fit a sine or cosine function to the empirical standard deviations to make such an adjustment for heterogeneity. The averages and the standard deviations of the final residuals at different points were essentially zero and one, respectively (fig. 2c; table 1). Hence, the residuals at each of the 12 points exhibited white noise properties.

\section{Correlations}

Since the time direction was absent in the residuals, the correlation between seasonally adjusted observations at any two points was a function of the distance between them, but not the direction. Thus, the maximum distance was 6 months (the distance between November and February, for example, is not 9 months, but rather 2 , since the direction in time was irrelevant). The empirical correlations were calculated between pair-wise residuals at different points, i.e. residuals available in the same patients at two time points. In this way, we accounted for intrapatient correlations. In figure 3 , the empirical correlations are plotted against the distance. For larger distances, correlations were lower, as expected. The natural choice of the correlation function was an exponential one:

$$
\rho_{k l}=b_{1} \exp \left(-b_{2} h\right),
$$

where $b_{1}$ and $b_{2}$ are constants to be estimated from empirical correlations, and $h$ is a distance between points where observations in vectors $R_{k}$ and $R_{l}$ were made, $k, l=$ $1, \ldots, 12$. The parameters of the fitted exponential correlation function (fig. 3) were estimated to be $b_{1}=0.85$ and $b_{2}=0.06$ (both significantly different from zero). The correlation function decayed almost linearly with increasing distance and seemed to describe data well.

\section{Conditional Prediction}

The $95 \%$ PI is defined as [20]:

$$
E\left(D_{i k} / D_{i l}\right) \pm 1.96 \sqrt{\operatorname{var}\left(D_{i k} / D_{i l}\right)} \text {. }
$$

Because we had logarithmically transformed the data before estimating the model, the prediction and both ends of the PI had to be back-transformed using the exponential function. Hence, the final prediction for a patient $i$ at time point $k$ given the observation at point $l, k, l$ $=1, \ldots, 12$, would be $\exp \left\{S_{k}+\sigma_{k}\left(D_{i l}-S_{l}\right)\right\}$, with PI

$$
\exp \left\{\left(S_{k}+\sigma_{k}\left(D_{i l}-S_{l}\right)\right) \pm 1.96 \sqrt{\sigma_{k}^{2}\left(1-\rho_{k l}^{2}\right)}\right\} .
$$

Clearly, the extent of variation at the month of observation does not influence the uncertainty in the prediction. It is only the level of uncertainty at the month of prediction and the correlation between the observation and prediction time points that contribute to the width of the PI. The longer away from the prediction time point the observation takes place, the lower will be the correlation $\rho_{k l}$, increasing the factor $1-\rho_{k l}^{2}$, which extends the PI. For uncorrelated observations, the correlation coefficient between two time points would be zero, and the component $\sigma_{k}^{2}\left(1-\rho_{k l}^{2}\right)$ controlling the width of the PI becomes simply the variance at the prediction point $\sigma_{k}^{2}$. Since the square of the correlation coefficient $\rho_{k l}^{2}$ is always a non-negative number less or equal to one, the $\sigma_{k}^{2}\left(1-\rho_{k l}^{2}\right) \leq \sigma_{k}^{2}$, and hence it is always smaller than in the case of uncorrelated observations.

We note that the predicted value at the month where the observation was made is equal to the actual observa- 
Fig. 4. Example of model performance when predicting $25(\mathrm{OH}) \mathrm{D}$ level for 2 imaginary patients, one with a measured level of $100 \mathrm{nmol} / \mathrm{l}$ in August (a) and one with a measured level of $40 \mathrm{nmol} / \mathrm{l}$ in April (b) with the corresponding 95\% PI (as dashed line).
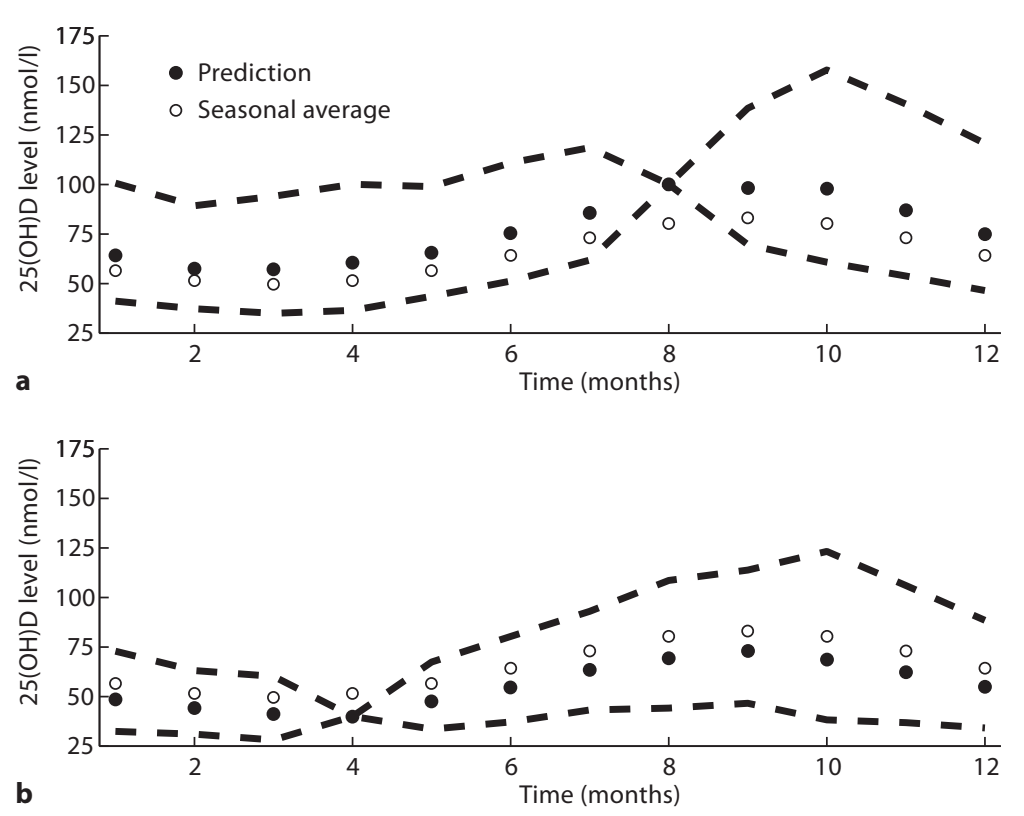

tion, while the variance at the month of observation is zero and thus the length of the PI is also zero.

\section{Example}

The proposed model allowed us to predict the concentration of $25(\mathrm{OH}) \mathrm{D}$ for a certain patient at any month with the corresponding PI by only using the information about the month of observation and the measured level of $25(\mathrm{OH}) \mathrm{D}$ at that particular month. The most uncertain prediction (widest PI) will likely be obtained in October, while the narrowest PI is expected in February, reflecting the largest and the smallest variation in these months, respectively (table 2). The differences in standard deviations will become more apparent after back-transformation. The PI will also be influenced by the correlation between the points of observation and prediction.

The components used in the prediction are tabulated in table 3. The values of the mean, $S_{k}$, were found from a seasonal component by inserting an appropriate $k$ value (first column). The variances, $\sigma_{k}^{2}$, are squares of standard deviations,

$$
\sqrt{S D_{B}^{2}+S D_{W}^{2}}
$$

to be found in table 2 for each point. The correlations, $\rho_{k l}$, for all unique distances are given in the last two columns.
Table 3. Tabulated values of the seasonal component $\left(S_{k}\right)$ and correlations $\left(\rho_{k l}\right)$ used in the prediction of $\log 25(\mathrm{OH}) \mathrm{D}$ levels

\begin{tabular}{llll}
\hline Month & $S_{k}$ & $h$ & $\rho_{k l}$ \\
\hline January & 4.03 & 1 & 0.80 \\
February & 3.94 & & \\
March & 3.90 & 2 & 0.75 \\
April & 3.94 & & \\
May & 4.03 & 3 & 0.71 \\
June & 4.16 & & \\
July & 4.29 & 4 & 0.66 \\
August & 4.39 & 5 & \\
September & 4.42 & & 0.62 \\
October & 4.39 & 6 & 0.59 \\
November & 4.29 & & \\
December & 4.16 & & \\
\hline
\end{tabular}

$h=$ distance (months) between the observation and prediction points.

Figure 4 illustrates the performance of the model for 2 imaginary patients, one with a measured $25(\mathrm{OH}) \mathrm{D}$ level of $100 \mathrm{nmol} / \mathrm{l}$ in August, and one with a measured $25(\mathrm{OH}) \mathrm{D}$ level of $40 \mathrm{nmol} / \mathrm{l}$ in April. Clearly, the first patient remained always above the seasonal average, while the second patient remained below seasonal average, as predicted by the model. The PIs became wider the farer 
Fig. 5. Validation of the model for 2 patients not used in the estimation. The seasonal average, predicted, and observed values are presented together with the corresponding 95\% PI (as dashed lines).
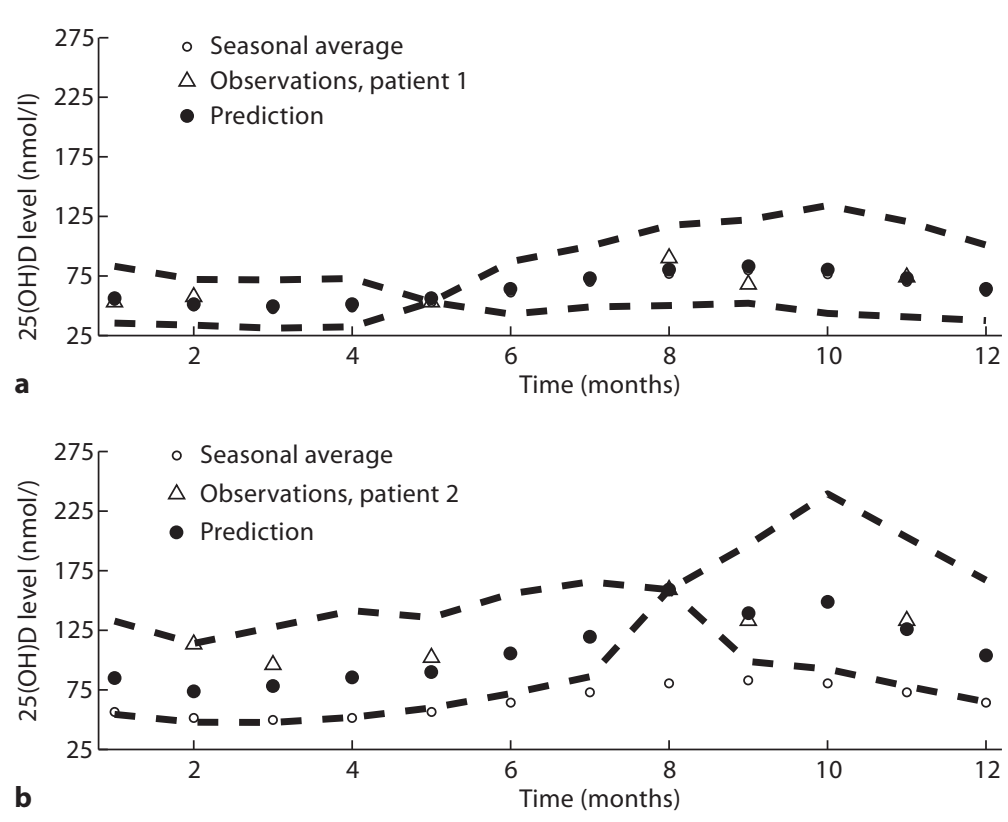

from the observation point the prediction was made, reflecting increasing uncertainty.

Our model was implemented in a spreadsheet (suppl. material) and can be used as a calculator for the prediction of 25(OH)D levels for an individual patient. Figure 4 is produced by the calculator by only inserting the time of observation and the measurement made for a patient.

\section{Validation}

Before estimating the model, the data on 2 patients were left out of the analysis and were later used for model validation. 25(OH)D values for these 2 patients were available at 6 months. As we expected the prediction to be less precise for patients with values far apart the average, we selected one patient who followed the estimated seasonal average closely and another with levels well above the estimated average. To validate the model, we entered one 'observed' value into the model and evaluated how well the model predicted values at other points of observation. For the 'average patient', all predicted values were within the PI. For the patient with observations higher than the average, the model performed a bit worse (one, sometimes two, observations were outside the PI), especially when the 'observed' value was close to the average (fig. 5).

Modelling and Prediction of 25(OH)D Levels in MS Patients

\section{Discussion}

Seasonal fluctuation of 25(OH)D levels [21], and also the corresponding incidence of rickets [22], has been known for decades. Nevertheless, current guidelines do not give any advice on how to incorporate seasonal fluctuation in the interpretation of $25(\mathrm{OH}) \mathrm{D}$ values [23], and the optimal level of $25(\mathrm{OH}) \mathrm{D}$ is extensively debated without specification of the timing of measurement [24]. Thus, a serum level of $100 \mathrm{nmol} / \mathrm{l}$ regardless of the season has been suggested as optimal for primary prophylaxis against MS [25]. One likely explanation for this paradox is the paucity of longitudinal data with repeated $25(\mathrm{OH}) \mathrm{D}$ assessments in individual subjects. The level of $25(\mathrm{OH}) \mathrm{D}$ has been shown to be relatively stable in individual patients when measured with an interval of several years, and baseline levels have been demonstrated to be significantly correlated with values at the subsequent $3,6,9$, and 12 months [26]. To our knowledge, the present study is the first to incorporate inter- and intra-individual variation in a model of the seasonal fluctuation of $25(\mathrm{OH}) \mathrm{D}$, thereby providing a tool for the prediction of vitamin $\mathrm{D}$ levels throughout the year based on one single measurement.

Our results show that the estimated seasonal average is clearly not sufficient for predicting an individual patient's level throughout a year. Indeed, we observed a sub-

Neuroepidemiology 2012;39:84-93 
stantial clustering effect at the individual level which was well captured by the model together with the seasonal fluctuations. Even $36.6 \%$ of the total variability could be accounted for by sources of intra-individual variations. By incorporating the information from repeated measurements into the model, we obtain a PI which always is narrower than in the case of uncorrelated observations. The observed clustering effect was rather strong, resulting in correlations between different points well above 0.5. The smallest reduction of width of PI for log $25(\mathrm{OH}) \mathrm{D}$ due to repeated measurements in our population was estimated to be $21 \%$ as compared to the uncorrelated observations.

The inter-individual differences in $\log 25(\mathrm{OH}) \mathrm{D}$ reflected $63.4 \%$ of the total variability. This percentage differed between months. The lowest total variability and inter-individual variability were observed in February, implying that the most representative $25(\mathrm{OH}) \mathrm{D}$ levels in MS patients in Norway are measured in February. Even though the correlation between the time points of observation and prediction plays a role in determining the width of the PI, the variance remains the main factor. According to the model, the narrowest PI was achieved in February, corresponding to the lowest variation in this period, and could be up to $26 \%$ narrower than the PI at other months. Our cohort was rather homogeneous regarding disease duration, disability, and treatment. Nevertheless, further inclusion of patient characteristics, e.g. gender, age, disability status, vitamin $\mathrm{D}$ supplement use, and sun exposure, would likely produce more homogeneous data (smaller inter-individual variations) and reduce the PI, but would require a larger cohort. The application of the presented principles of modelling in another, preferentially larger, data set could further validate our results.

In addition to MS, a poor vitamin D status has been associated with numerous other extra-skeletal diseases, including cancer, cardiovascular diseases, systemic lupus erythematosus, diabetes mellitus, and rheumatoid arthritis [27]. Both seasonal averages and the level of variation will likely differ in other populations and geographical regions with different climates. Accordingly, it has recently been shown that seasonal fluctuation of 25(OH)D levels differs between East Asian, European, and South Asian young adults living in Toronto, Ont., Canada [28]. The model presented here was estimated on data from relatively newly diagnosed Norwegian relapsing-remitting MS patients with a low level of disability. The estimates are therefore valid for this particular population and may thus differ in patients with more ad- vanced disability who are likely to spend less time outdoors exposed to UVB radiation. The principles of modelling and prediction should, however, upon adjustment of the estimates, be applicable also to other populations. The estimation procedure is described in detail in this paper and can be applied on any data set containing $25(\mathrm{OH}) \mathrm{D}$ levels. Increasing numbers of observations per patient reduce PI, and the use of repeated measurements per patient is therefore advantageous. The time resolution does not change the model structure. However, a more exact adjustment of the time resolution than every month would result in a reduced PI, and vice versa.

Both the expressions for the prediction and PI are presented, and the online calculator (suppl. material) is designed for the case of one available observation for an individual patient. In the case where more than one observation are available, mathematical expressions would be somewhat more cumbersome but altogether easily implementable.

\section{Funding}

This study was supported by Merck-Serono, Pronova Biocare, Bayer Schering, The Western Norway Regional Health Authority, Odd Fellow Research Foundation for multiple sclerosis, Kåre Wærner's Legacy, and The Norwegian MS Society.

\section{Disclosure Statement}

J. Šaltytė Benth reports no disclosures.

K.-M. Myhr has received honoraria for lecturing, participation in pharmaceutical company-sponsored clinical trials, and travel support from Bayer Schering, Biogen Idec, Novartis, Merck-Serono, and Sanofi Aventis.

K.I. Løken-Amsrud has received travel support and an unrestricted research grant from Biogen Idec and travel support from Merck Serono.

A.G. Beiske has received travel support from Merck Serono and honoraria for lecturing from Novartis and Biogen Idec.

K.S. Bjerve reports no disclosures.

H. Hovdal has participated in pharmaceutical company-sponsored clinical trials and received travel support from Merck Serono, Biogen Idec, and Bayer Schering.

R. Midgard has received honoraria for lecturing, participation in pharmaceutical company-sponsored clinical trials, and travel support from Bayer Schering, Biogen Idec, Novartis, Merck-Serono, and Sanofi Aventis.

T. Holmøy has received speaker honoraria and travel support from Sanofi Aventis, Biogen Idec, Bayer Schering, Novartis, and Merck Serono. 


\section{References}

$>1$ Smolders J, Moen SM, Damoiseaux J, Huitinga I, Holmøy T: Vitamin D in the healthy and inflamed central nervous system: access and function. J Neurol Sci 2011;311:37-43.

$>2$ Munger KL, Levin LI, Hollis BW, Howard NS, Ascherio A: Serum 25-hydroxyvitamin $D$ levels and risk of multiple sclerosis. JAMA 2006;296:2832-2838.

$\checkmark 3$ Ascherio A, Munger KL, Simon KC: Vitamin $D$ and multiple sclerosis. Lancet Neurol 2010;9:599-612.

4 Mowry EM, Krupp LB, Milazzo M, et al: Vitamin D status is associated with relapse rate in pediatric-onset multiple sclerosis. Ann Neurol 2010;67:618-624.

$>5$ Smolders J, Menheere P, Kessels A, Damoiseaux J, Hupperts R: Association of vitamin $\mathrm{D}$ metabolite levels with relapse rate and disability in multiple sclerosis. Mult Scler 2008; 14:1220-1224.

-6 Simpson S Jr, Taylor B, Blizzard L, et al: Higher 25-hydroxyvitamin D is associated with lower relapse risk in multiple sclerosis. Ann Neurol 2010;68:193-203.

$>7$ Soilu-Hänninen M, Airas L, Mononen I, Heikkila A, Viljanen M, Hanninen A: 25-Hydroxyvitamin D levels in serum at the onset of multiple sclerosis. Mult Scler 2005; 11:266-271.

$>8$ Soilu-Hänninen M, Laaksonen M, Laitinen I, Eralinna JP, Lilius EM, Mononen I: A longitudinal study of serum 25-hydroxyvitamin D and intact parathyroid hormone levels indicate the importance of vitamin D and calcium homeostasis regulation in multiple sclerosis. J Neurol Neurosurg Psychiatry 2008;79:152-157.

$>9$ Holick MF: Vitamin D status: measurement, interpretation, and clinical application. Ann Epidemiol 2009;19:73-78.

$>10$ Holick MF: Vitamin D deficiency. N Engl J Med 2007;357:266-281.

$\checkmark 11$ Webb AR, Kline L, Holick MF: Influence of season and latitude on the cutaneous synthesis of vitamin D3: exposure to winter sunlight in Boston and Edmonton will not promote vitamin D3 synthesis in human skin. J Clin Endocrinol Metab 1988;67:373-378.
12 Ahn J, Yu K, Stolzenberg-Solomon R, Simon KC, McCullough ML, Gallicchio L, Jacobs EJ, Ascherio A, Helzlsouer K, Jacobs KB, Li Q, Weinstein SJ, Purdue M, Virtamo J, Horst R, Wheeler W, Chanock S, Hunter DJ, Hayes RB, Kraft P, Albanes D: Genome-wide association study of circulating vitamin D levels. Hum Mol Genet 2010;19:2739-2745.

13 Orton SM, Morris AP, Herrera BM, Ramagopalan SV, Lincoln MR, Chao MJ, Vieth R, Sadovnick AD, Ebers GC: Evidence for genetic regulation of vitamin $\mathrm{D}$ status in twins with multiple sclerosis. Am J Clin Nutr 2008;88: 441-447.

14 Torkildsen $\varnothing$, Wergeland S, Bakke SJ, Beiske AG, Bjerve KS, Hovdal H, Midgard R, Lilleås F, Pedersen T, Bjørnarå B, Dalene F, Kleveland G, Schepel J, Olsen IC, Myhr KM: Omega-3 Fatty Acid Treatment in Multiple Sclerosis (OFAMS study): a randomised, doubleblind, placebo-controlled trial. Arch Neurol 2012, E-pub ahead of print.

15 Løken-Amsrud KI, Holmøy T, Bakke SJ, Beiske AG, Bjerve KS, Bjørnarå BT, Hovdal H, Lilleås F, Midgard R, Pedersen T, Šaltytė Benth J, Sandvik L, Torkildsen $\varnothing$, Wergeland S, Myhr KM: Vitamin D and disease activity in multiple sclerosis before and during inter feron- $\beta$ treatment. Neurology 2012, E-pub ahead of print.

16 Stamp TC, Round JM: Seasonal changes in human plasma levels of 25-hydroxyvitamin D. Nature 1974;247:563-565.

17 McLaughlin M, Raggatt PR, Fairney A, et al: Seasonal variations in serum 25-hydroxycholecalciferol in healthy people. Lancet 1974; 1:536-538.

18 Anderson TW: An Introduction to Multivariate Statistical Analysis, ed 3, revised. Hoboken, NJ, Wiley \& Sons, Wiley Series in Probability and Statistics, 2003.
19 van der Mei IAF, Ponsonby AL, Dwyer T, Blizzard L, Taylor BV, Kilpatrick T, Butzkueven H, McMichael AJ: Vitamin D levels in people with multiple sclerosis and community controls in Tasmania, Australia. J Neurol 2007;254:581-590.

20 Chatfield C: Time Series Forecasting. Boca Raton, London, New York, Washington, DC, Chapman\&Hall/CRC, 2000.

-21 Gupta MM, Round JM, Stamp TC: Spontaneous cure of vitamin-D deficiency in Asians during summer in Britain. Lancet 1974; 1: 586-588.

-22 Arneil GC, Crosbie JC: Infantile rickets returns to Glasgow. Lancet 1963;2:423-425.

-23 Holick MF, Binkley NC, Bischoff-Ferrari HA, Gordon CM, Hanley DA, Heaney RP, Murad MH, Weaver CM: Evaluation, treatment, and prevention of vitamin $\mathrm{D}$ deficiency: an Endocrine Society clinical practice guideline. J Clin Endocrinol Metab 2011;96: 1911-1930.

24 Grant WB, Juzeniene A, Moan JE: Review Article: Health benefit of increased serum $25(\mathrm{OH}) \mathrm{D}$ levels from oral intake and ultraviolet-B irradiance in the Nordic countries. Scand J Public Health 2011;39:70-78.

25 Simon KC, Munger KL, Ascherio A: XVI European Charcot Foundation lecture: nutrition and environment, can MS be prevented? J Neurol Sci 2011;311:1-8.

-26 Jorde R, Sneve M, Hutchinson M, Emaus N, Figenschau Y, Grimnes G: Tracking of serum 25-hydroxyvitamin D levels during 14 years in a population-based study and during 12 months in an intervention study. Am J Epidemiol 2010;171:903-908.

-27 Moreno LA, Valtuena J, Perez-Lopez F, Gonzalez-Gross M: Health effects related to low vitamin D concentrations: beyond bone metabolism. Ann Nutr Metab 2011;59:22-27.

-28 Gozdzik A, Barta JL, Weir A, Cole DE, Vieth R, Whiting SJ, Parra EJ: Serum 25-hydroxyvitamin D concentrations fluctuate seasonally in young adults of diverse ancestry living in Toronto. J Nutr 2010;140:22132220 . 